\section{Entwicklung und Diagnostik des Sprachverständnisses bei Deutschschweizer Kindern}

\author{
Erich Hartmann, Barbara Rindlisbacher, \\ Christoph Till, Julia Winkes \\ Universität Freiburg/Schweiz
}

\section{Problemstellung}

Das Sprachverstehen ist ein wichtiger Aspekt des kindlichen Spracherwerbs, dem im Hinblick auf den schulischen und beruflichen Erfolg große Bedeutung zukommt. Ebenso ist unbestritten, dass Kinder mit Problemen in der rezeptiven Sprachverarbeitung - z.B. im Kontext von Sprachentwicklungsstörungen - ein hohes Risiko für eine negative Lern- und psychosoziale Entwicklung tragen (vgl. Amorosa/Noterdaeme 2003; Kannengieser 2009; Thelen 2013). Umso wichtiger ist es, Sprachverständnisschwierigkeiten frühzeitig zu erkennen, um den betroffenen Kindern angemessene Fördermaßnahmen zukommen zu lassen (vgl. Clark u.a. 2007; Mathieu 2007; Buschmann/ Jooss 2011).

Eine Herausforderung für die logopädische Diagnostik ist, dass sich Sprachverstehensprozesse einer direkten Beobachtung entziehen. Zu deren Einschätzung muss daher auf Verhaltensindikatoren zurückgegriffen werden. In freien Erhebungssituationen kann das Sprachverstehen nur global und unbefriedigend eingeschätzt werden. Zum einen ist eine gezielte Überprüfung relevanter Sprachstrukturen nicht möglich, zum anderen sind viele Kinder mit Verständnisschwierigkeiten fähig, Alltagssituationen mithilfe erlernter Strategien relativ erfolgreich zu meistern, weswegen ihr Nichtverstehen dem Laien (z.B. Eltern) nicht immer bewusst wird (vgl. Amorosa/Noterdaeme 2003; Möller u. a. 2008; Buschmann/Jooss 2011). Selbst Experten können (subtilere) Auffälligkeiten des Sprachverstehens übersehen, sofern nicht eine vertiefte Diagnostik mittels standardisierter und altersnormierter Tests durchgeführt wird (vgl. Kannengieser 2009; Thelen 2013).

Aufgrund lückenhafter Grundlagenforschung zum Spracherwerb Deutschschweizer Kinder sowie eines Mangels an (aktuellen, validen) schweizerdeutschen Diagnostikinstrumenten, greifen hiesige Logopädinnen und Logopäden in der Praxis gezwungenermaßen auf Tests zurück, welche an Hochdeutsch sprechenden Kindern normiert wurden. Solche Instrumente, wie etwa der TROG-D Test zur Überprüfung des Grammatikverständnisses (Fox 2013), werden in der Deutschschweiz entweder in der Originalversion (Hochdeutsch) oder häufig auch auf Schweizerdeutsch durchgeführt, wobei die Übersetzung in diesem Fall informell durch die Logopädin für den jeweiligen Dialekt vorgenommen wird (vgl. Mathieu 2000).

Beide Vorgehensweisen sind beim aktuellen Forschungsstand mit Schwierigkeiten und Unsicherheiten behaftet: Zum einen ist es bei einer hochdeutschen Durchführung fraglich, ob die deutschen Testnormen ungeprüft auf Kinder aus dem schweizerdeutschen Raum übertragen werden können, zumal sich Letztere in Bezug auf ihre Sprachsozialisation bzw. -entwicklung (Erwerb eines schweizerdeutschen Dialekts, späterer Kontakt mit dem Hochdeutschen) von Kindern aus Deutschland unterscheiden. Um mögliche diagnostische Fehleinschätzungen bzw. Unterschätzungen von Schweizer Kindern in (hoch-)deutschen Sprachtests identifizieren und vermeiden zu können, sollten die Leistungen von Schweizer Kindern bei solchen Instrumenten vermehrt überprüft und mit den Originalnormen verglichen werden.

Zum anderen muss bei einer schweizerdeutschen Testdurchführung bedacht werden, dass die zu überprüfenden Strukturen im Hochdeutschen und im Schweizer Dialekt teilweise differieren können (z.B. Kasusmarkierung) bzw. eine direkte Übersetzung der originalen Testitems ins Schweizerdeutsche nicht durchgängig möglich ist. Wird aber eine schweizerdeutsche Übersetzung des Tests - mit informell abgeänderten oder weggelassenen Prüfaufgaben - genutzt, so beeinträchtigt dies nicht nur die inhaltliche Validität und ggf. die Zuverlässigkeit der Erhebung, sondern einmal mehr auch die Angemessenheit der Anwendbarkeit von deutschen Normen auf Sprachleistungen von Deutschschweizer Kindern.

Vor diesem Hintergrund sind zur Klärung offener diagnostischer Fragen empirische Studien 
angezeigt, welche die Leistungen von Schweizer Kindern u.a. in Sprachverständnistests in hochdeutscher Version versus Dialektvariante systematisch vergleichen. Die Ergebnisse solcher Untersuchungen sind nicht nur aus der Sicht der Grundlagenforschung von Interesse, sondern dürften auch für evidenzbasierte Entscheidungen in der logopädischen Praxis relevant und hilfreich sein.

\section{Fragestellungen und Annahmen}

Das vorzustellende Projekt adressiert eine Forschungslücke und intendiert die empirische Beantwortung folgender Fragen:

1. Unterscheiden sich die Leistungen von Deutschschweizer Kindern in einem standardisierten Sprachverständnistest (bzw. im TROG-D) in Abhängigkeit von der Präsentationsbedingung „Hochdeutsch“vs. „Schweizerdeutsch“?

2. Welche (quantitativen und qualitativen) Veränderungen im Sprachverstehen zeichnen sich vom Kindergarten bis Ende der 3. Klasse ab?

3. Inwieweit differieren Deutschschweizer Kinder und deutsche Kinder in ihren hochdeutschen Sprachverständnisleistungen? Sind die deutschen Testnormen für die Diagnostik mit Deutschschweizer Kindern adäquat?

4. Wie präsentiert sich das Sprachverständnis von Kindern mit besonderen Sprachlernbedingungen (Mehrsprachigkeit, Sprachstörung u. a.) im Vergleich zu Kontrollkindern?

Die zentralen Annahmen der Studie lauten, dass a) Schweizer Kinder die Dialektversion des TROG$D$ besser bewältigen als die deutsche Originalversion, wobei sich der Unterschied über die Jahre reduzieren sollte, b) Schweizer Kinder im Vergleich zu deutschen Kindern deutlich schwächere hochdeutsche Verständnisleistungen zeigen und c) Kinder nicht (Schweizer-)Deutscher Muttersprache bzw. Kinder mit Sprachstörungen ein geringeres Sprachverständnis zeigen als sprachunauffällige Kontrollkinder.

Sollte Erwartung b) bestätigt werden, so wäre dies ein Argument gegen die Anwendung der deutschen Originalnormen in der logopädischen Praxis der Deutschschweiz bzw. ein Argument für eine eigene Normierung der schweizerdeutschen resp. auch der hochdeutschen Testversion an Kindern aus der Deutschschweiz.

\section{Methodisches Vorgehen}

Die explizierten Fragestellungen sollen im Rahmen einer Querschnittsstudie mit einer repräsentativen Stichprobe von insgesamt 600 Berner Regelschulkindern (Kindergarten, 1.-3. Klasse, jeweils gegen Ende Schuljahr) geklärt werden. Alle Probanden bearbeiten randomisiert eine schweizerdeutsche und eine hochdeutsche (originale) Version des TROG-D (Fox 2013).

Beim TROG-D handelt es sich um ein aktuelles, an deutschen Kindern normiertes Diagnostikinstrument, welches auch in der Schweiz häufig zur Anwendung gelangt. Der TROG-D überprüft das Sprachverständnis auf Satzebene mit verschiedenen linguistischen Strukturen. Für das Projekt wird mit dem Departement für Literatur und Linguistik der Universität Freiburg/Schweiz (Mehrsprachigkeitsinstitut) eine möglichst äquivalente schweizerdeutsche (berndeutsche) Testversion erstellt.

Die standardisierte Präsentation der Items beider Versionen geschieht mittels eigens zu diesem Zweck erstellter Audio-Aufnahmen, um die Durchführungsobjektivität zu gewährleisten (vgl. Deevy 2009).

Zur Datenanalyse werden neben deskriptiven Möglichkeiten je nach Fragestellung und Stichprobenmerkmalen verschiedene inferenzstatistische Verfahren (u.a. Varianzanalyse, t-Test für abhängige Stichproben, Effektstärkeberechnung) genutzt.

\section{Ausblick}

Zum Zeitpunkt der Manuskripterstellung liegt die Bewilligung des Kantons Bern zur Durchführung der Studie vor, und ein Drittmittelantrag wurde vom Deutschschweizer Logopädinnenund Logopädenverband DLV bewilligt.

Als weitere Projektschritte sind neben der Stichprobenrekrutierung die Entwicklung der schwei- 
zerdeutschen Testversion, die Erstellung der Audio-Aufnahmen sowie die Auswahl und Schulung von Testleiterinnen vorgesehen, damit die Untersuchung wie geplant Ende Schuljahr 2014/15 durchgeführt werden kann. Über die Realisierung und die Ergebnisse des Projekts soll zu gegebener
Zeit in einer fachwissenschaftlichen Zeitschrift informiert werden.

Weitere Informationen und Literaturangaben können eingeholt werden bei christoph.till@ unifr.ch 